\title{
Biogene Wasserstoffproduktion und deren Analytik
}

\author{
Nora Haufe $^{1}$, Felix Krujatz ${ }^{2}$, Jost Weber ${ }^{2}$, Sara Grützner ${ }^{3}$, Matthias Schelter ${ }^{4}$, Jens Zosef', Michael \\ Mertig ${ }^{1,4}$ \\ ${ }^{1}$ Physikalische Chemie, Mess- und Sensortechnik, Technische Universität Dresden, 01062 Dresden, \\ nora.haufe@tu-dresden.de \\ 2 Bioverfahrenstechnik, Technische Universität Dresden, 01062 Dresden \\ ${ }^{3}$ Wasserstoff- und Kernenergietechnik, Technische Universität Dresden, 01062 Dresden \\ ${ }^{4}$ Kurt-Schwabe-Institut für Mess- und Sensortechnik e.V. Meinsberg, Kurt-Schwabe-Straße 4, \\ 04736 Waldheim
}

\begin{abstract}
Kurzfassung:
Wir zeigen in unserem Ansatz ein alternatives Verfahren der Bio-Wasserstoffproduktion. Die biologische Aktivität von Algen und die von Bakterien wird in einem Tandemprozess kombiniert genutzt. Um Wasserstoff zu erzeugen, muss das System nur mit Wasser und Licht "gefüttert“ werden. Somit handelt es sich bei dem produzierten Wasserstoff um einen Biokraftstoff.

Um unser Ziel zu realisieren, werden zwei Bioreaktoren so miteinander verbunden, dass die Reaktionsprodukte aus dem Behälter der Entstehung zu dem der Nutzung diffundieren können. Einer der Reaktoren beinhaltet die Algen, der andere die Bakterien.

Die Algen erzeugen Kohlenwasserstoffe, mit denen die Bakterien dann gefüttert werden. Zum Aufbau der Kohlenwasserstoffe wird nur Kohlenstoffdioxid $\left(\mathrm{CO}_{2}\right)$ und Licht benötigt. Der zweite Reaktor beinhaltet die Bakterien, Rhodobacter sphaeroides, die aus den Kohlenwasserstoffen Wasserstoff und Kohlenstoffdioxid erzeugen. Letzterer wird in einer Art Kreislauf wieder den Algen zugeführt und von innen verstoffwechselt. Durch dieses Zusammenspiel komplementieren sich beide Systeme.

Ein grundlegender Teil der Entwicklung ist das Überwachen einzelner Prozessparameter des Tandemregimes, was durch eine zuverlässige Online-Gasanalytik realisiert werden soll. Unser Beitrag diskutiert verschiedene Möglichkeiten Wasserstoff gasförmig und in gelöster Form sowie weitere Gase zu detektieren.
\end{abstract}

Schlagworte: Biowasserstoff, Gasanalytik, Biokraftstoff, Wasserstoffextraktion.

\section{Problemstellung}

Aufgrund des steigenden Energiebedarfes ist es nicht nur wichtig Ressourcen zu schonen, sondern auch neuartige Ansätze zu deren Gewinnung zu liefern. Viele Arbeitsgruppen forschen deshalb an alternativen Herstellungswegen. Im Rahmen einer Nachwuchsforschergruppe wird hier der Fokus auf Wasserstoff als universeller Energieträger gelegt. Dabei wird ein $\mathrm{CO}_{2}$-neutrales, bionisches Verfahren genutzt. Durch Kombination mit einer Brennstoffzelle sind mobile sowie dezentrale/lokale Anwendungen möglich.

\section{Lösungsweg und Ergebnisse}

In einer Co-Kultivierung von Algen und Purpurbakterien wird Wasserstoff phototrop erzeugt. Die Algen liefern dabei die von den
Bakterien benötigten Nährstoffe, $\left(\mathrm{C}_{x} \mathrm{H}_{\mathrm{y}} \mathrm{O}_{z}\right)_{n}$. Bei Stickstoffmangel wird von den Bakterien Wasserstoff und Kohlenstoffdioxid produziert. Letzterer soll im Kreislauf wieder den Algen zugeführt werden, sodass als Bilanz nur Wasserstoff und Sauerstoff entstehen.

Abbildung 1 veranschaulicht die beiden zugrunde liegenden Reaktionsschritte.

Um den Reaktor zu konzipieren und das System anschließend optimieren zu können, ist eine genaue Analytik des produzierten und der gelösten Gase notwendig. Wasserstoff, Kohlenstoffdioxid, Stickstoff und Sauerstoff sind dabei primär von Interesse. Besondere Herausforderungen stellen dabei auch die Sicherheit (explosive Gasgemische) sowie die große Mobilität des Wasserstoffs dar. 
Für die Bestimmung des Wasserstoffgehalts im Produktgas sowie gelöst in der Bakteriensuspension wird folgender analytischer Aufbau eingesetzt: (I) Trennung durch Gaschromatographie und (II) coulometrische Detektion mit einer Elektrolysezelle.

Voruntersuchungen wurden mit einem Wärmeleitfähigkeitsdetektor durchgeführt. In Abbildung 2 ist eine Messung des produzierten Gases aus dem Bakterienreaktor gezeigt. Eine Extraktion der Gelöstgase, die bei der Untersuchung des gelösten Wasserstoffs vorgeschaltet werden muss, wird nach dem Prinzip von Schelter et al. [1, 2] realisiert.

\section{Danksagung}

Das Projekt wird durch den Europäischen Sozialfond (ESF) und die Sächsische Aufbaubank (SAB) gefördert.

\section{References}

[1] M. Schelter, J. Zosel, W. Oelßner, U. Guth, M. Mertig, Gelöstgas-Analytik in biogenen Medien, 10. Dresdner Sensor-Symposium, 313-316 (2011); doi: 10.5162/10dss2011/16.7

[2] M. Schelter, Untersuchungen von Messverfahren zur Bestimmung gelöster Gase in Medien zur Biogasproduktion, Masterarbeit, Technische Universität Dresden (2011)

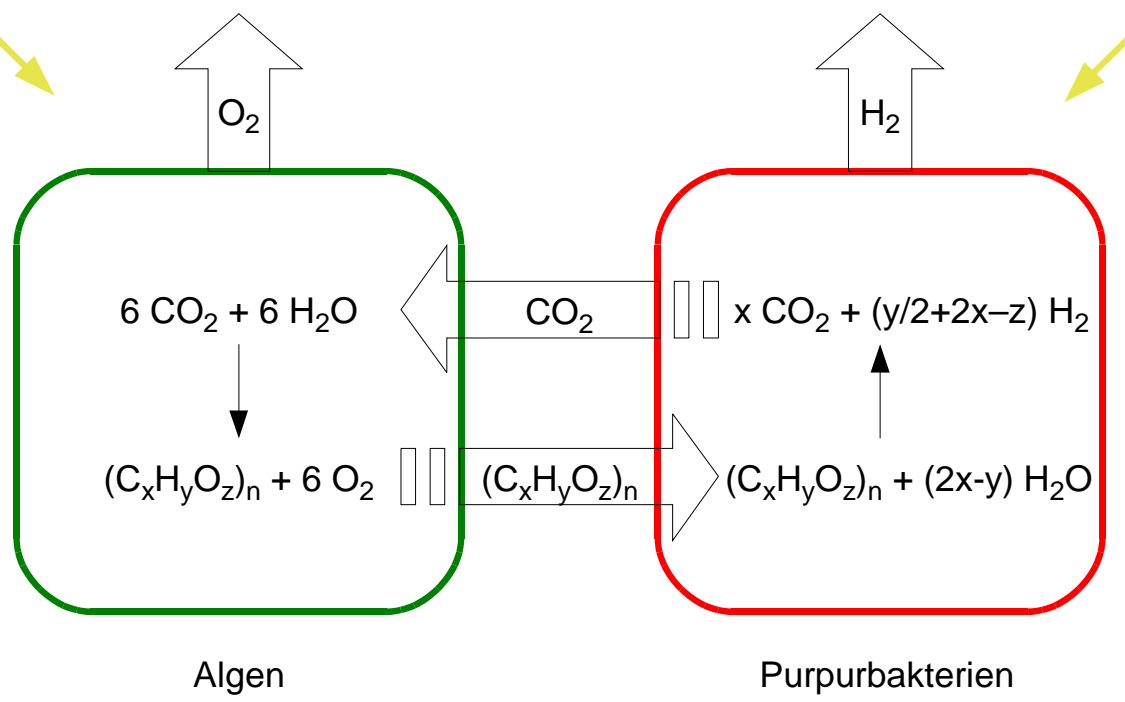

Fig. 1. Prozessübersicht.

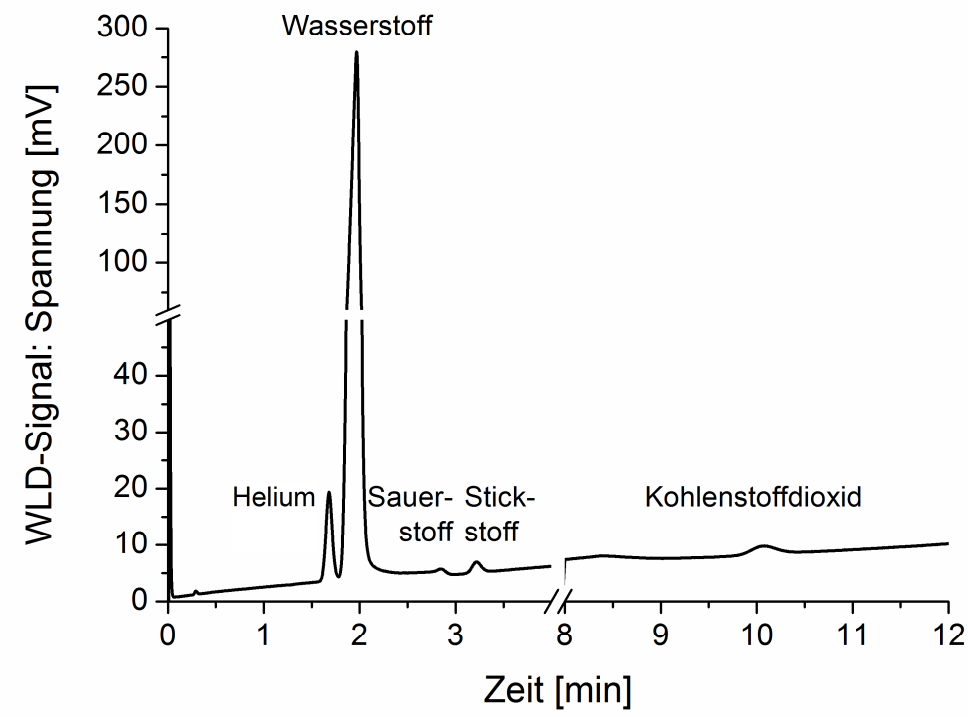

Fig. 2. Analyse des produzierten Gasgemischs mit den Signalen für $\mathrm{He}, \mathrm{H}_{2}, \mathrm{~N}_{2}, \mathrm{O}_{2}$ und $\mathrm{CO}_{2}$ (v.l.n.r.). 